\title{
Measurement of Turbulent Intensity Distributions of a Cylinder Wake
}

\author{
Deog Hee Doh**, Gyeong Rae Cho*, Kyeong Rok Moon** and Yong Beom Cho**
}

\begin{abstract}
Turbulence properties of a cylinder wake $(\mathrm{d}=10 \mathrm{~mm})$ have been investigated with a new volume PTV algorithm. The measurement system consists of two-high-cameras $(1 \mathrm{k} \times 1 \mathrm{k})$, a Nd-Yag laser and a host computer. A fitness function representing three-dimensional coherency has been adopted to sort out spurious vectors. A hybrid fitness function representing the relations between the fitness and the threedimensional shortest distances constructed by the two collinears of the two cameras has been also adopted. The constructed algorithm has been employed for the measurements of the cylinder wakes. The Reynolds numbers tested in this paper are 360, 540, 720, 900, 1080 and 1260. More than 10,000 instantaneous 3D vectors have been obtained by the constructed system. The volumetric distributions of the turbulence intensities (for u', v', w') indicate that clearly different patterns for all Reynolds numbers and imply that a regular pattern (like a similarity rule) for the turbulent properties exists.
\end{abstract}

Key Words: Turbulent intensity, Cylinder wake, Volume PTV

\section{Introduction}

Accurate quantification of turbulence properties in the near-wake region is of a great interest concerning the physical analysis and the turbulence modeling of unsteady separated flows past bluff bodies. The main objectives of the present study are to provide a detailed evaluation of the mean and fluctuating turbulent quantities in the near-wake field and in the vicinity of the wake region of a cylinder wake.

Monkewitz $^{(1)}$ showed the existence of a local absolute instability in the wake at a Reynolds number of $\operatorname{Re}=25$. However Williamson ${ }^{(2)}$ showed that a von Karman vortex street appears at a Reynolds number around 49. At a critical Reynolds number of $\mathrm{Re}=194$ the wake flow becomes three-dimensional. The first three-dimensional instability that appears is the socalled "mode A instability" that is characterized by a spanwise wave-length of around 3D-4D, where D is the diameter of the cylinder. This mode is the result

\footnotetext{
'Korea Maritime Univ., Division of Mechanical \& Energy Systems Eng.,

E-mail : doh@hhu.ac.kr

**LG Electronics, Home Appliance, Changwon, Korea
}

of an elliptic instability and it scales on the primary vortex core, which is the larger physical structure in the flow. Around $\mathrm{Re}=240$ the instability changes from mode A to mode B. Mode B is characterized by a reduction in size of the spanwise wave-length by a factor of three. This instability scales on the braid shear layer which is the smaller physical structure of the flow ${ }^{(3)(4)}$. In the subcritical range - for Reynolds numbers between $\mathrm{Re}=1000$ and $\mathrm{Re}=200,000$ - the flow in the vicinity of the cylinder is entirely laminar and transition happens somewhere in the free shear layer downstream of the cylinder. In this study, turbulence properties near the wake of a circular cylinder were investigated by using a newly constructed Volume PTV.

In the meantime, there have been many challengeable studies for obtaining spatial distributions of velocity vectors of the fluid flows. Arroyo and Greated ${ }^{(5)}$ and Kähler and Kompenhans ${ }^{(6)}$ showed the capability of the Stereo-PIV (SPIV) for probing three-dimensional properties of the flow fields. Hinsch ${ }^{(7)}$ and Chan et al. ${ }^{(8)}$ showed possibility of quantification of the complex flows by using Holography-PIV(HPIV). Digital-HolographicPIV was firstly constructed by Coëtmellec et $a l .^{(9)}$. Very recently, Kim and Lee ${ }^{(10)}$ constructed in-line 
digital holographic PIV(DHPIV) by introducing a high-definition camera $(1 \mathrm{~K} \times 1 \mathrm{~K})$. Their results showed that the recovery ratio from the whole particles in the measurement volume was about $65 \%$ when the particle density was $25 / \mathrm{mm}^{3}$. This means that the whole identifiable particle numbers are about 8,500 among the 13,000 particles appeared in the measurement volume. Sung and $\mathrm{Yoo}^{(11)}$ introduced a phase-averaging PIV technique for obtaining threedimensional wake structures of a circular cylinder wake by the use of 2D-PIV. They suggested how to overcome the limits of the spatial resolution of the 3Dmeasurement techniques using their 2D-PIV results. Recently, a Tomographic PIV (Volume PIV) has been suggested by Scarano et al. ${ }^{(12)}$ and employed to the measurements of the cylinder wake.

The purpose of the study is to evaluate spatial distributions of the turbulent properties of a circular cylinder wake by using a newly constructed Volume PTV.

\section{Experimental Setup}

Fig. 1 shows the overall measurement system. A cylinder $(\mathrm{D}=10 \mathrm{~mm})$ was installed into a water channel $(1100 \mathrm{~mm} \times 300 \mathrm{~mm} \times 300 \mathrm{~mm})$. Two digital cameras (Kodak ES1.0, $1 \mathrm{k} \times 1 \mathrm{k}$ ) were used for volumetric measurements and installed as seen in Fig. 1. A NdYag laser $(120 \mathrm{~mJ}, 15 \mathrm{~Hz})$ was used for flow visualiza-

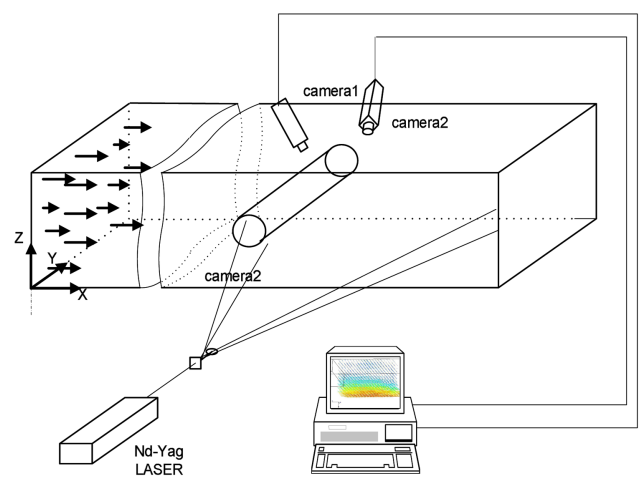

Fig. 1. Experimental set-up. tions. A logic controller (Labsmith, LC880) was used for synchronizations with the cameras and the Nd-Yag laser. In order to calculate the camera parameters, a calibration plate on which the grid lines were installed with $4 \mathrm{~mm} \times 4 \mathrm{~mm}$ distances was used. This plate was traversed in the measurement volume to the cameras' viewing direction. The Reynolds numbers tested were 360, 540, 720, 900, 1080 and 1260. The measurement volume was set to $60 \mathrm{~mm} \times 70 \mathrm{~mm} \times 20 \mathrm{~mm}$.

\subsection{Principle of Volume PTV}

The principle of the newly constructed volume PTV (particle tracking velocimetry) follows two steps. Firstly, camera calibration is carried out for the two cameras, through which the relation between the physical coordinate of the measurement volume and the photographical coordinate of the camera images was obtained. Next, finding (tracking) process was made, in which the same particle pairs between the two time consecutive image frames were obtained.

\subsubsection{Particle Image Matching}

In order to attain three-dimensional measurements with two cameras, it is necessary to know their camera parameters. The 10-parameter method ${ }^{(13)(14)}$ was used to obtain them. In this method, 10 parameters (6 exterior parameters: dis, $\alpha, \beta, \gamma, m_{x}, m_{y}, 4$ interior parameters: $\left.c_{x}, c_{y}, k_{1}, k_{2}\right)$ were otained. $(\alpha, \beta, \gamma$,$) represents$ the tilting angles of the axes of the photographic coordinates against the absolute axes. The collinear equation for every point between the two coordinates is expressed as Eq. (1). $c_{x}$ and $c_{y}$ are the focal distances for $\mathrm{x}$ and $\mathrm{y}$ components of the coordinate. $\Delta \mathrm{x}$ and $\Delta \mathrm{y}$ are the lens distortions as expressed as Eq. (2).

$$
\begin{aligned}
& x=c_{s} \frac{Y_{m}-m_{x}}{\sqrt{d i s^{2}-m_{x}^{2}-m_{y}^{2}}-Z_{m}}+\Delta x \\
& y=c_{y} \frac{Y_{m}-m_{y}}{\sqrt{d i s^{2}-m_{x}^{2}-m_{y}^{2}}-Z_{m}}+\Delta y \\
& \Delta x=(x / r) \times\left(k_{1} r^{2}+k_{2} r^{4}\right), \Delta y=(x / r) \times\left(k_{1} r^{2}+k_{2} r^{4}\right), \\
& r=\sqrt{x^{2}+y^{2}}
\end{aligned}
$$




$$
\begin{aligned}
& F=c_{x} \frac{X_{m}-m_{x}}{\sqrt{d i s^{2}-m_{x}^{2}-m_{y}^{2}}-Z_{m}}-(x-\Delta x)=0 \\
& G=c_{y} \frac{X_{m}-m_{y}}{\sqrt{d i s^{2}-m_{x}^{2}-m_{y}^{2}}-Z_{m}}-(x-\Delta y)=0
\end{aligned}
$$

The Eq. (2) can be converted to Eq. (3). Explanations on the symbols in the equations and the calculation process for the camera parameters are explained well in the reference ${ }^{(13)}$.

\subsubsection{Tracking Algorithm}

Fig. 2 shows the tracking algorithm of the Volume PTV. Vector tracking algorithm is performed as follows. (1) Obtain the 2-dimensional vectors for each camera within a certain displacement. Here, PM (particle movement) has been set to a certain pixel value according to the flow speed (Fig. 3). This procedure is called as time matching. (2) Find candidate of particle pairs on the image of camera 2. Here, Epipolar line has been used to find the candidate particles on the image of the camera 2. To save calculation time, a certain search area has been set as shown in Fig. 4. The particle pairs in this area are regarded as the candidates. For the particle ' $n 1$ ' and ' $\mathrm{n} 2$ ', the same procedure for finding the candidates is

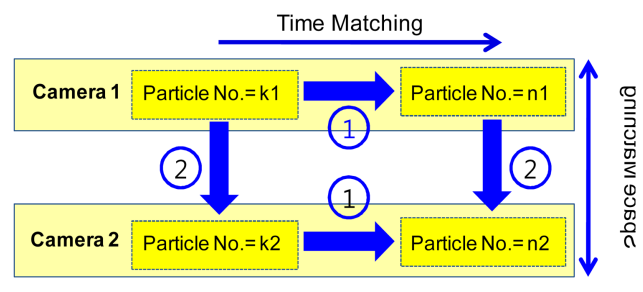

Fig. 2. Overall procedure for vector acquisitions.

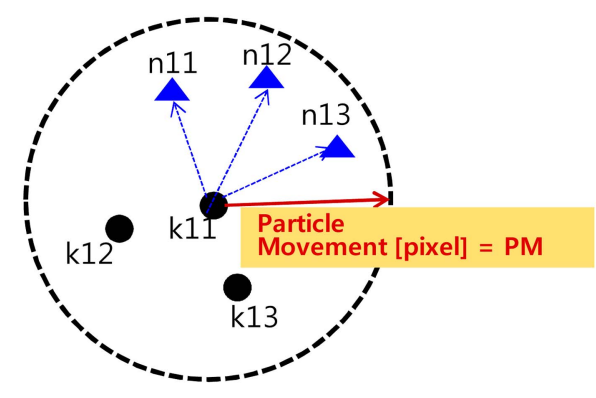

Fig. 3. Definition of particle movement [PM].

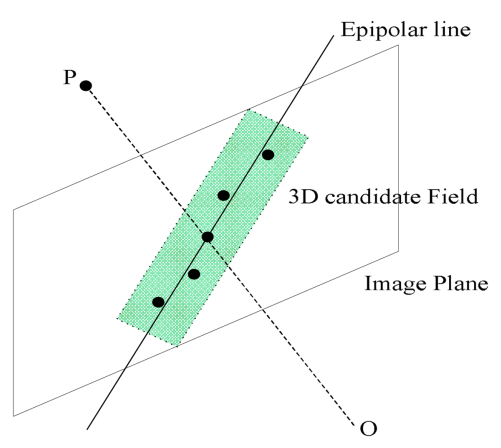

Fig. 4. Epipolar line and a search area.

made. Once the candidate $(\mathrm{k} 2)$ of the particle image of the camera 2 for the initial point $(\mathrm{k} 1)$ are found, the terminal point of the $2 \mathrm{D}$ vector is automatically decided since the $2 \mathrm{D}$ vectors had been already obtained. If the candidate for particle ' $\mathrm{n} 1$ ' is found within the Epipolar search area, the particle set of $(k 1, n 1$, $\mathrm{k} 2, \mathrm{n} 2$ ) is sorted into the candidate group. (3) For the whole particles in the images of the camera 1 and the camera 2, the candidate group database is constructed. (4) For the candidate group database, a particle neighborhood value $[\mathrm{PN}]$ is set to a certain value $[\mathrm{mm}]$. Within this PN, a vector fitness [VF] is calculated using Eq. (4). $u_{i}$ represents the whole vectors within PN except the target vector itself, and $u_{o}$ represents the mean value of the whole vectors within PN except the target vector itself. (5) Lastly, a sigmoid-like hybrid fitness function as shown in Fig. 6 is used for sorting out the most probable candidate from the candidate group database. VF values in Eq. (4) are again fitted to the

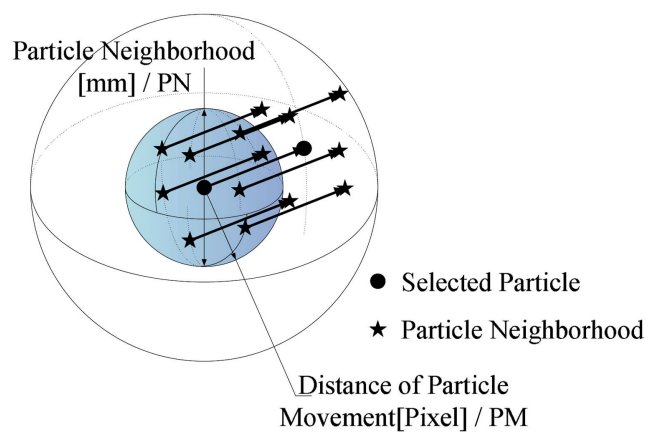

Fig. 5. Definition of particle neighborhood [PN]. 


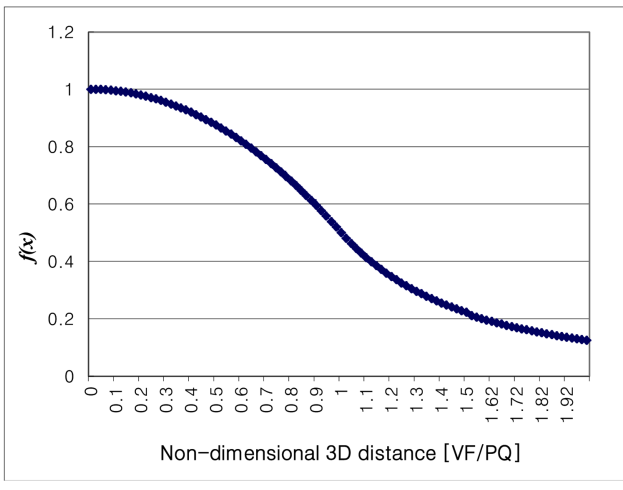

Fig. 6. Hybrid fitness function used for sorting the most probable candidate from the candidate group database.

$f(x)$ curve according to Eq. (5). VF values were nondimensionalised with PQ. PQ value was set to $0.5 \mathrm{~mm}$. Using PN and PM values implies finding a coherency of the neighborhood particles. It was confirmed that the optimal parameters were $\mathrm{PM}=8$ pixel, $\mathrm{PN}=5 \mathrm{~mm}$ and $\mathrm{VF}=0.3$.

$$
\begin{aligned}
& \text { Vector Fitness }[V F]=\frac{\sum\left|u_{i}-u_{o}\right|}{\sum u_{i}} \\
& f(x)=\left\{\begin{array}{lll}
-0.5 x^{2}+1, & \text { at } & (0 \leq x \leq 1) \\
0.5 x^{2}, & \text { at } & (x \geq 1)
\end{array}\right.
\end{aligned}
$$

\section{Results and Discussions}

Fig. 7 shows the obtained instantaneous 3D vectors at $\operatorname{Re}=360$. More than 10,000 vectors were obtained. This number is very meaningful because the number was recovered from the identified particles in one image. This implies that the vector recovery is almost close to $80 \sim 90 \%$ of the particles seen in the image. Fig. 8 shows the obtained instantaneous 3D vector distributions interpolated onto $40 \times 40 \times 6$ grids when the Reynolds number is 360 .

Fig. 9 shows the turbulence properties of the wake at $\mathrm{Re}=360$. The turbulent intensities of $\mathrm{w}$ ' component were larger than those of u' and v' values. For all Reynolds number cases, $\left|w^{\prime}\right|>\left|u^{\prime}\right|>\left|v^{\prime}\right|$ was satisfied, and

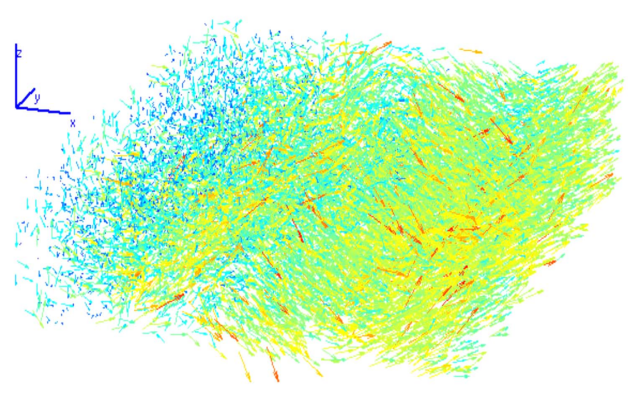

Fig. 7. Instantaneous $3 \mathrm{D}$ vector field $(\operatorname{Re}=360)$.

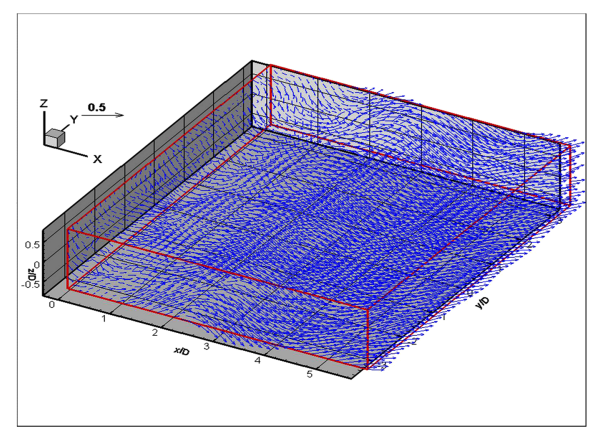

Fig. 8. Instantaneous interpolated vectors $(\mathrm{Re}=360)$.

each value was 2 times higher than others. w' distribution had a strong relation with those of the turbulent kinetic energy, which implies that w' component largely influences the turbulent properties of the wake.

Fig. 10 and Fig. 11 indicate $\left|u^{\prime}\right| U_{o}$ and $\left|v^{\prime}\right| / U_{o}$ distributions, respectively for Reynolds 540, 720, 900, and 1080. With increase of Reynolds numbers, the maximum value of $\left|v^{\prime}\right| U_{o}$ increased, which implies that $v^{\prime}$ component (spanwise fluctuations) mainly contributes to the energy balancing of the turbulent structure at high Reynolds number. $\left|u^{\prime}\right| U_{o}$ value was maximum at $\operatorname{Re}=720$. At low Reynolds numbers, v' component showed B-mode structure while u' component showed B-mode structure $^{(15)}$ at high Reynolds numbers. Regardless of Reynolds numbers, the distribution of the u' component showed a well organized structure near wake region, which implies that the energy cascade of the u' component occurs with regular patterns at near wake and irregular 


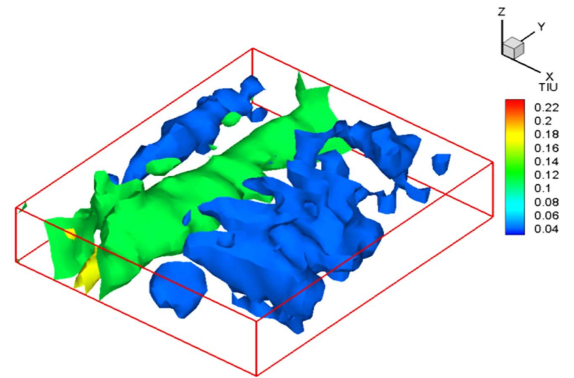

(a) $\left|u^{\prime}\right| / U_{o}$

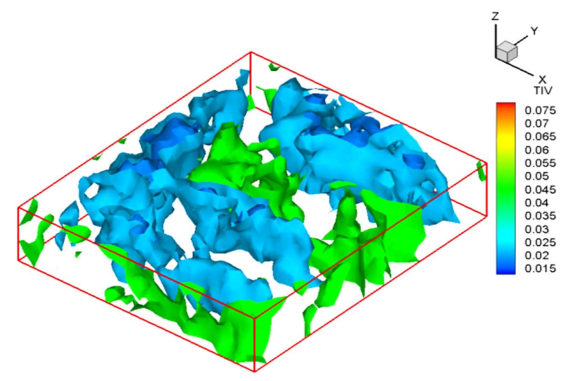

(b) $\left|v^{\prime}\right| / U_{o}$

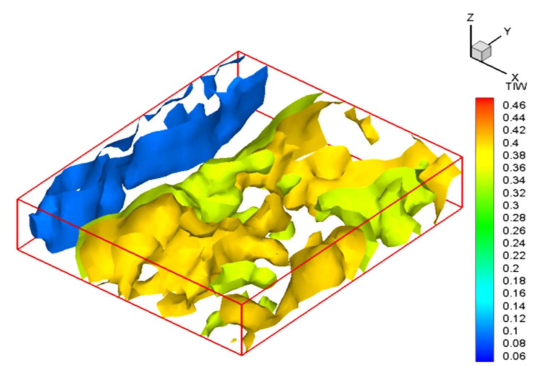

(c) $\left|w^{\prime}\right| / U_{o}$

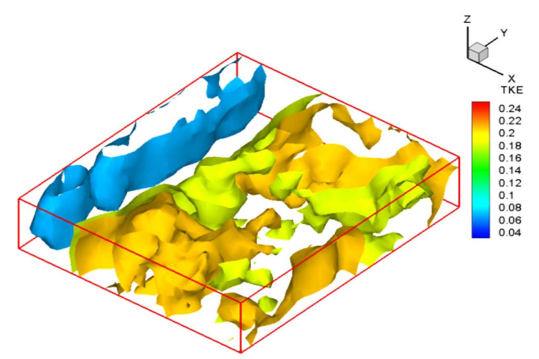

(d) $\sqrt{u^{\prime 2}+v^{\prime 2}+w^{\prime 2}} / U_{o}$

Fig. 9. Turbulence properties $(\mathrm{Re}=360)$.

pattern at downstream. The distributions of $\left|u^{\prime}\right| U_{o}$, $\left|v^{\prime}\right| / U_{o}$ and $\left|w^{\prime}\right| / U_{o}$ were spanwise within the region of

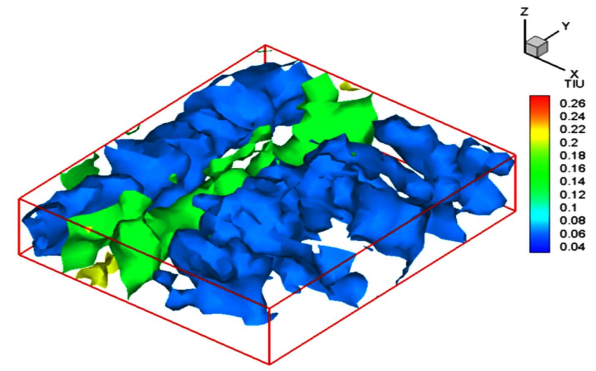

(a) at $\mathrm{Re}=540$

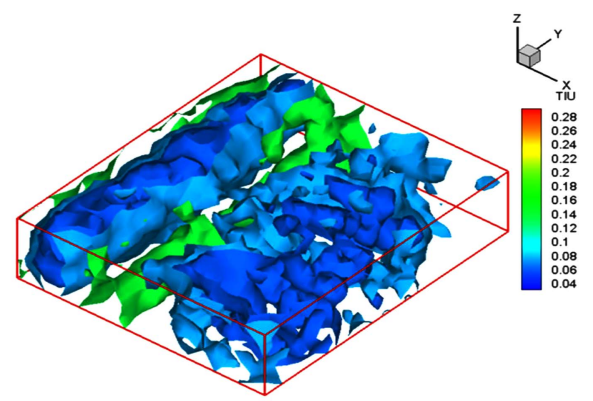

(b) at $\mathrm{Re}=720$

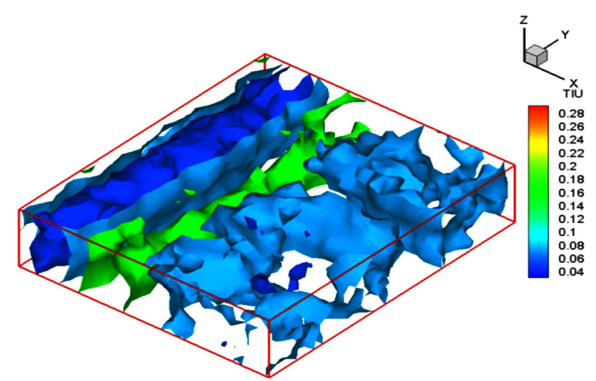

(c) at $\mathrm{Re}=900$

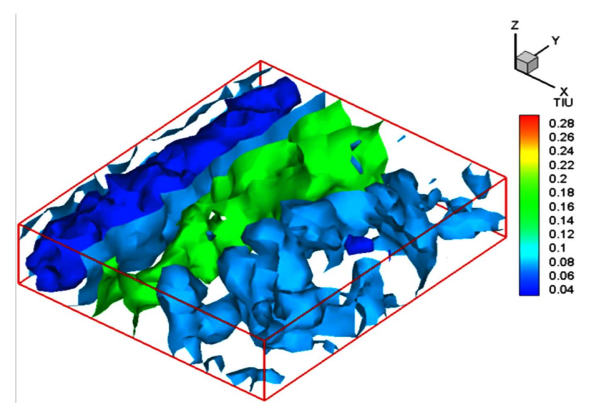

(d) at $\operatorname{Re}=1080$

Fig. 10. Spatial distribution of $\left|u^{\prime}\right| U_{o}$.

$1.5 \mathrm{~d}(\mathrm{~d}=10 \mathrm{~mm})$ from the center of the cylinder, and were streamwise distributions at the area over $1.5 \mathrm{~d}$. 


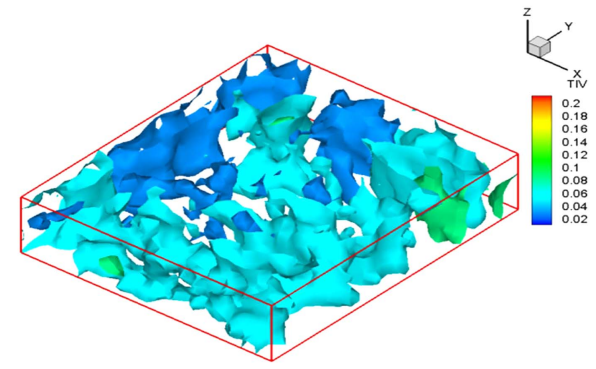

(a) at $\mathrm{Re}=540$

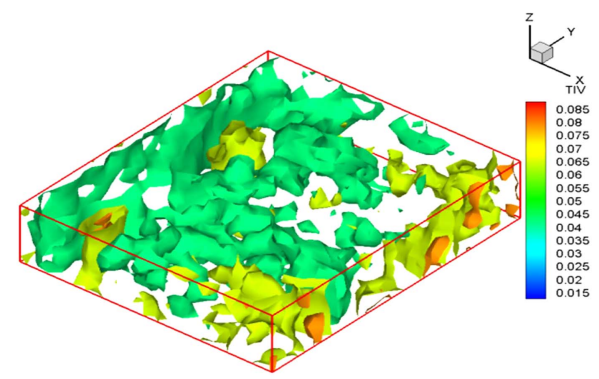

(b) at $\mathrm{Re}=720$

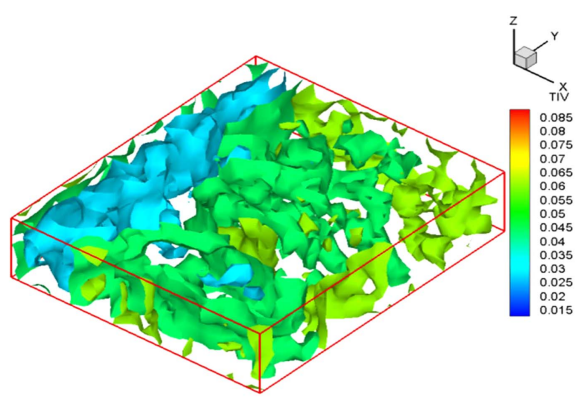

(c) at $\mathrm{Re}=900$

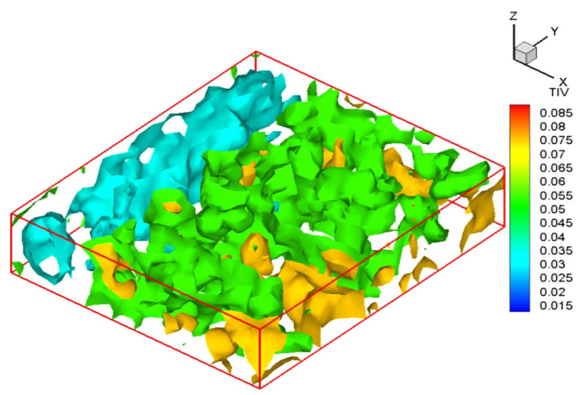

(d) at $\mathrm{Re}=1080$

Fig. 11. Spatial distribution of $\left|v^{\prime}\right| U_{o}$.

\section{Summaries}

Turbulent properties of the circular cylinder wake have been investigated by using a newly constructed Volume PTV. For successful measurements, a hybrid fitness function and a coherency fitness function have been adopted. More than 10,000 instantaneous 3D velocity vectors could be obtained by the constructed Volume PTV system. Obtained velocity vector fields showed reasonable physical properties on the circular cylinder wake.

The optimal parameters used the measurements were PM [particle movements] $=8$ pixels, PN [particle neighborhood] $=5 \mathrm{~mm}$ and $\mathrm{VF}$ [vector fitness] $=0.3$ under the threshold value, PQ value, were set at $0.5 \mathrm{~mm}$.

From the experiments, several outcomes were obtained as follows. For all Reynolds number cases, $\left|w^{\prime}\right|>\left|u^{\prime}\right|>\left|v^{\prime}\right|$ was satisfied, and each value was 2 times higher than others. The turbulent intensity distribution of $\mathrm{w}^{\prime}$ has a strong relation with that of the turbulent kinetic energy $\sqrt{u^{\prime 2}+v^{\prime 2}+w^{\prime 2}} / U_{o}$, which implies that $\mathrm{w}$ ' component largely influences the turbulent properties of the wake. From the analyses on the vertical structure, it showed that the distance between the second primary vortex and the third primary vortex was 1.5 times longer than that of the distance between the first primary vortex and the second primary vortex.

\section{Acknowledgements}

This work was supported by the National Research Foundation Grant funded by the Korean Government (MEST) (No. 2008-0060153). This work (Grants No. 00046109) was supported by Business for Academicindustrial Cooperative establishments funded by Korea Small and Medium Business Administration in 2011. The content of this paper had already been published in the proceedings of the $6^{\text {th }}$ International Symposium on Turbulence and Shear Flow Phenomena, and partly in proceedings of the $8^{\text {th }}$ International Symposium on PIV.

\section{References}

1) Monkewitz, P.A., 1988, "The Absolute and Convective 
Nature of Instability in Two-Dimensional Wakes at Low Reynolds Numbers", Phys. Fluids, Vol. 31, pp. 999-1006.

2) Williamson, C.H.K., 1996a, "Vortex Dynamics in the Cylinder Wake", Ann. Rev. Fluid Mech., Vol. 28, pp. 477-539.

3) Williamson, C.H.K., 1996b, "Three Dimensional Wake Transition", J. Fluid Mech., Vol. 328, pp. 345407.

4) Thompson, M., Hourigan, M., and Sheridan, J., 1996. "Three Dimensional Instabilities in the Wake of a Circular Cylinder", Exp. Therm. Fluid Sci., Vol. 12, pp. 190-196.

5) Arroyo, M. P., and Greated, C. A., 1991, "Stereoscopic Particle Image Velocimetry", Meas. Sci Technol, Vol. 2, pp. 1181-1186.

6) Kähler, C. J., and Kompenhans, J., 2000, "Fundamentals of Multiple Plane Stereo Particle Image Velocimetry", Exp Fluids, Suppl, pp. S70-S77.

7) Hinsch, K. D., 2002, "Holographic Particle Image Velocimetry", Meas Sci Technol, Vol. 13, pp. R61R72.

8) Chan, VSS., Koek, WD., Barnhart, D.H., Bhattacharya, N., Braat, JJM. \& Westerweel, J. 2004. "Application of Holography to Fluid Flow Measurements using Bacteriorhodopsin", Meas Sci Technol, Vol. 15, pp. 647-655.

9) Coëtmellec, S., Buraga-Lefebvre, C., Lebrun, D., and Özkul, C. 2001, "Application of In-line Digital Holography to Multiple Plane Velocimetry", Meas Sci Technol, Vol. 12, pp.1392-1397.

10) Kim, S., and Lee, S.J., 2008 "Effect of Particle Number Density in In-line Digital Holographic Particle Velocimetry", Exp. in Fluids, Vol. 44. pp.623631.

11) Sung J., and Yoo, J. Y., 2001, "Three-Dimensional Phase-Averaging of Time-Resolved PIV Measurement Data", Meas. Sci. Technol., Vol. 12, pp. 655662.

12) Scarano, F., Elsinga, G.E., Bocci, E, and van Oudheusden, B.W. 2006 "Investigation of 3-D Coherent Structures in the Turbulent Cylinder Wake using Tomo-PIV", CD-ROM Proc. of 13th Intl' Symp. on Appl. of Laser Tech. to Fluid Mech., Lisbon, 26-29 June.

13) Doh, D.H., Kim, D.H., Cho, K.R., Cho, Y.B., Saga, T., and Kobayashi, T. 2002, "Development of Genetic Algorithm based 3D-PTV Technique”, Journal of Visualization, Vol. 5, No.3, pp. 243-254.

14) Doh, D. H., Hwang, T. G., and Saga, T., 2004, “3DPTV Measurements of the Wake of a Sphere", Meas Sci Technol, Vol. 15(6), pp.1059-1066.

15) Brede, M., Eckelmann, H., and Rockwell, D., 1996, "On Secondary Vortices in the Cylinder Wake", Phys. Fluids, Vol. 8, pp. 2117-2124. 